\title{
New Frontiers in the Medical Therapy of Hepatocellular Carcinoma
}

\author{
Claudia Angela Maria Fulgenzi ${ }^{a, b}$ Antonio D'Alessio ${ }^{a, c}$ Thomas Talbot ${ }^{a}$ \\ Alessandra Gennari $^{d}$ Mark R. Openshaw ${ }^{a}$ Coskun O. Demirtas ${ }^{\mathrm{e}}$ \\ Alessio Cortellini ${ }^{a, f}$ David J. Pinato ${ }^{a, d}$ \\ ${ }^{a}$ Department of Surgery \& Cancer, Imperial College London, Hammersmith Hospital, London, UK; ${ }^{\circ}$ Department of \\ Medical Oncology, University Campus Bio-Medico, Rome, Italy; ' Department of Biomedical Sciences, Humanitas

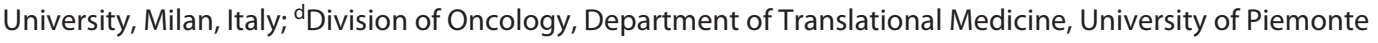

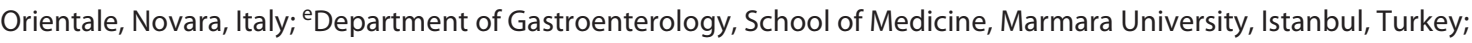 \\ fDepartment of Biotechnology and Applied Clinical Sciences, University of L'Aquila, L'Aquila, Italy
}

\section{Keywords}

Hepatocellular carcinoma · Immunotherapy · Systemic treatments

\begin{abstract}
Background: Hepatocellular carcinoma (HCC) is the most common primary liver tumor, and it rates fourth as a cause of cancer-related death. The presence of underlying liver disease and poor chemosensitivity pose major treatment challenges in the management of HCC. However, in the last few years, the therapeutic scenario has substantially changed, and immunotherapy in the form of immune checkpoint inhibitors (ICPIs) has become an essential therapeutic strategy in this field. Summary: After controversial results of monotherapy, ICPIs have been mainly investigated in association with antiangiogenic agents or as dual checkpoint inhibition. The combination of atezolizumab plus bevacizumab has become the new therapeutic standard for unresectable HCC. Currently, a number of ICPI-based combinations are being studied in phase III clinical trials as front-line therapy for advanced HCC, with growing interest in integration of early-
\end{abstract}

karger@karger.com www.karger.com/che

Karger $\stackrel{\text { ' }}{5}$

BOPEN ACCESS
(C) 2022 The Author(s)

Published by S. Karger AG, Basel

This is an Open Access article licensed under the Creative Commons Attribution-NonCommercial-4.0 International License (CC BY-NC) (http://www.karger.com/Services/OpenAccessLicense), applicable to the online version of the article only. Usage and distribution for commercial purposes requires written permission. stage disease management in the form of adjuvant or neoadjuvant therapies. With most of the trials investigating ICPIs as first-line treatment, the second-line scenario relies mainly on tyrosine kinase inhibitors, which however have not been formally trialed after ICPIs. Key Messages: In this review, we summarize the main therapeutic advances in the systemic management of $\mathrm{HCC}$ focusing on the most relevant ongoing trials. We also discuss the main issues arising from a such rapidly evolving field including therapeutic sequencing and patient stratification.

(c) 2022 The Author(s).

Published by S. Karger AG, Basel

\section{Introduction}

Hepatocellular carcinoma (HCC) is the most common primary liver cancer, and due to its intrinsic chemoresistance, it has historically been deprived of effective treatments [1]. Alongside scarcity of therapeutic options, the presence and severity of the underlying liver disease, which is an accompanying feature of almost all cases of HCC, contributes to worsen patient's prognosis 
[2] and requires a careful balance between treatment benefits and risk of hepatic decompensation [3]. Curative approaches are reserved to early stages (Barcelona Clinic Liver Cancer-BCLC 0/A), and they include surgical resection, local ablative techniques (radiofrequency ablation, cryoablation, microwave ablation, and percutaneous ethanol injection), and liver transplantation (OLT). The recurrence rate after surgery and ablation is still high [4], and liver transplantation is feasible only in a small percentage of patients falling into rigid criteria [5]. For patients with intermediate or advanced stage HCC, treatment is pursued with palliative intent. Transarterial chemoembolization (TACE) is recommended for intermediate stage (BCLC-B), and systemic treatments are the only option for advanced stage HCC (BCLC-C). Sorafenib is the first drug to show survival advantage over placebo in advanced stage HCC, and despite leading to a net improvement in overall survival (OS) of almost 3 months compared to placebo, it has remained the only available option for a decade [6]. Moreover, sorafenib has mainly a cytostatic action, and therefore its effect against tumor-related symptoms is limited and so is its capacity to downstage the disease [6]. After sorafenib approval, other tyrosine kinase inhibitors (TKIs) have been tested as first-line treatments with disappointing results until 2018, when lenvatinib was approved as first-line treatment for advanced HCC, following the results of the phase III noninferiority REFLECT trial [7]. After years of skepticism, mainly due to safety concerns regarding the delivery of immunotherapy in patients with hepatitis and underlying liver dysfunction, promising results from phase I/II trials paved the way to immunotherapy in HCC as early as 2017. Despite initial optimism, the first phase III trial testing nivolumab as first-line treatment in advanced HCC failed to show significant survival advantage over sorafenib [8], leading researchers to investigate the association of ICPIs with other agents, in order to augment efficacy and improve survival. In 2020, the combination of atezolizumab plus bevacizumab showed an OS advantage over sorafenib, and this resulted in FDA approval as front-line therapy for advanced HCC [9]. After encouraging evidence of activity in early phase studies, other combinations are currently being tested in phase III trials and, after a decade of disappointing results, are expected to further expand this therapeutic scenario of HCC. However, the availability of multiple therapeutic choices has raised other issues in the treatment landscape of HCC, with patient selection and definition of optimal treatment sequencing being the most relevant.

\section{Immune Checkpoint Inhibitor Monotherapy}

Immunotherapy has substantially changed the therapeutic landscape of different types of cancer in the last few years. In particular, immune checkpoint inhibition has become the most widely adopted strategy to activate the host immune system against cancerous cells [10] (shown in Fig. 1). Under physiological conditions, immune checkpoints prevent overactivation of immune cells against self-antigens and modulate immune response during inflammation. The inhibitory function of immune checkpoints is commonly exploited by tumor cells to avoid immune system attack [1]. Amongst the checkpoint molecules, programmed cell death 1 (PD-1) and cytotoxic-T-lymphocyte-associated protein 4 (CTLA-4) have emerged as clinical targets, and several specific antibodies are currently used in cancer medicine to prevent the interaction between PD-1 and CTLA-4 with their ligands (PD-L1/2 and B7-1/2, respectively) in order to restore T-cells' antitumor function [11].

HCC is a paradigmatic example of an inflammatory tumor, and in fact, it usually arises on the background of chronic liver inflammation which promotes an immune exhausted microenvironment. Furthermore, the liver is physiologically exposed to antigens through the gut, and this contributes to maintain an immune tolerant environment [12]. These characteristics provide the rationale to test immune checkpoint inhibitors (ICPIs) in HCC. However, the development of immunotherapy in HCC has been hampered by a number of factors including safety concerns over the delivery of immunotherapy in patients with hepatotropic viral infection and liver dysfunction, the lack of predictive biomarkers, and, most recently, lack of evidence for survival improvement both in first- and in second-line use of PD-1 inhibitors [13].

CTLA-4 is an immune checkpoint receptor expressed by regulatory and activated $\mathrm{T}$ cells, interacting with its ligands (B7-1 and B7-2) on antigen-presenting cells, and it inhibits T-cell activity. The pharmacological inhibition of CTLA- 4 has been exploited as an anticancer strategy in different malignancies, mainly in association with antiPD-1/PD-L1 drugs. In HCC, anti-CTLA-4 antibodies are not approved as monotherapy [14].

Tremelimumab is an anti-CTLA-4 monoclonal antibody, and it has been the first ICPI to be tested in a cohort of 20 patients with HCV-related HCC [15]. Overall, 17.6\% of patients had objective response, and a drop in circulating HCV-RNA levels was also described. Tremelimumab monotherapy was further tested in a cohort of a phase I/ II trial assessing the safety of durvalumab and tremelim- 


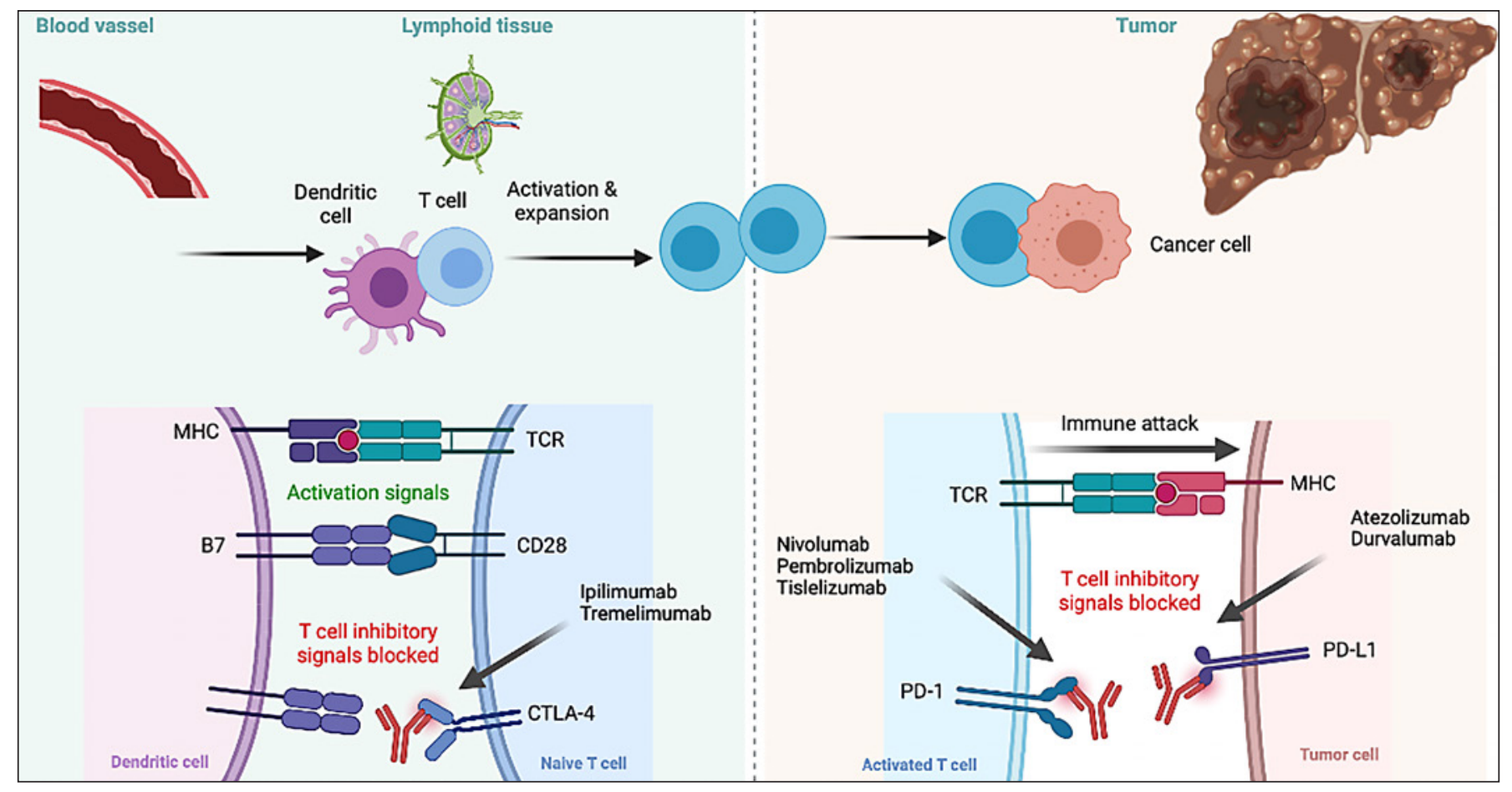

Fig. 1. Schematic representation of the immunologic mechanisms underlying the efficacy of the forerunner immune checkpoint inhibitors in HCC.

umab combination therapy after sorafenib [15]. In total, 69 patients were treated with tremelimumab $(750 \mathrm{mg}$ every 4 weeks $\times 7$ doses and then once every 12 weeks) within the trial. This cohort achieved an objective response rate (ORR) of $7.2 \%$, and an OS of 15.1 months which was higher than that reported by durvalumab monotherapy.

In 2017, results from the CheckMate-040 led to the FDA approval of nivolumab as second-line treatment for advanced HCC, in patients with well-preserved liver function (Child-Pugh class A). CheckMate-040 was a phase I/II trial which tested the safety and efficacy of nivolumab, a monoclonal IgG4 anti-PD-1 antibody, in previously treated HCC patients [16]. The trial was composed of an escalation and an expansion phase, and no safety concerns were raised during the first part. Nivolumab showed an ORR of $15 \%$ and $20 \%$ in the escalation and expansion cohorts, and an OS of 15 months in patients previously treated with sorafenib in the dose escalation cohort [16]. The following phase III CheckMate-459 trial compared nivolumab to sorafenib as first-line treatment, and despite leading to longer OS, it did not achieve the statistical significance per prespecified criteria according, among others, to the number and dimension of tumor nodules, age, and comorbidities [8]. Nivolumab therapy was well tolerated with a man- ageable toxicity profile both in phase I/II and phase III trials. In 2021, nivolumab lost FDA approval for the treatment of advanced HCC after failure to sorafenib following voluntary withdrawal by the manufacturer.

Pembrolizumab is another anti-PD-1 IgG4 monoclonal antibody which has been evaluated as monotherapy in advanced HCC. The phase II Keynote-224 trial enrolled 104 previously treated HCC patients ( $80 \%$ had progressed during sorafenib treatment and $20 \%$ were sorafenib-intolerant) to receive pembrolizumab monotherapy (200 $\mathrm{mg}$ every 3 weeks). Pembrolizumab therapy resulted in an ORR of $17 \%$ and an OS of 12.9 months, with an acceptable safety profile [17], leading to breakthrough designation by the FDA in 2018 as a second-line agent for advanced HCC previously treated with sorafenib. The efficacy of pembrolizumab versus placebo was investigated in patients previously treated with sorafenib, in the phase III Keynote-240 trial, which randomized 443 to receive pembrolizumab or placebo in a 2:1 ratio. Patients in the experimental arm reported a median OS of 13.9 months, compared to 10.6 of placebo (HR 0.78; 95\% CI: 0.61-0.99; $p=0.0238$ ). Despite the absolute benefit, the OS advantage derived from pembrolizumab did not reach the statistical significance in comparison with placebo $(p=0.0174)$. The ORR was 
higher in the experimental arm compared to placebo (18.2\% vs. $4.4 \%)$. The approval of second-line treatments (regorafenib and nivolumab) during trial accrual could have contributed to the overperformance of the placebo arm in terms of OS. Pembrolizumab has been also tested in Asian patients as second-line treatment after sorafenib in the phase III placebo-controlled III Keynote-394 trial (NCT03062358), and it has recently been announced to improve OS, progression-free survival (PFS), and ORR compared to placebo [18].

Durvalumab is another ICPI, an IgG1 monoclonal antibody targeting PD-L1. In HCC patients pretreated with sorafenib, durvalumab has been tested for safety and efficacy in a phase I/II basket trial, reporting an ORR of $10.3 \%$ and an OS of 13.2 months, with a surprisingly high OS of 19.3 months in HCV-infected subjects [19]. Similar results were described in the 104 patients who received durvalumab monotherapy (1,500 mg every 4 weeks) after sorafenib progression or intolerance, within the phase I/II trial assessing the safety of the combination of durvalumab and tremelimumab at different doses; in this case, there was not any difference according to the etiology [20].

The disappointing results of ICPI monotherapy in phase III trials highlight the urgent need for predictive factors to identify those patients who could respond to monotherapy and, at the same time, prompted researchers to investigate other strategies to overcome the intrinsic immunotherapy resistance of HCC. Currently, immune therapy in HCC is mainly delivered in association with antiangiogenic drugs or as dual checkpoint inhibition. RATIONALE 301 is the only ongoing phase III trial testing ICPI monotherapy compared to sorafenib in the first-line setting for advanced HCC [21]. The trial is currently randomizing patients to receive tislelizumab, an anti-PD-1 monoclonal antibody or sorafenib, and results are eagerly awaited.

\section{Combinations}

In 2020, the combination of atezolizumab plus bevacizumab was approved by the FDA as first-line treatment for advanced/unresectable HCC [9]. The approval was based on the results of the phase III open label randomized trial IMbrave 150 [22]. The trial randomized 501 patients in a $2: 1$ ratio to receive atezolizumab, an anti-PD-L1 monoclonal antibody (at 1,200 mg every 3 weeks), plus bevacizumab, an anti-vascular endothelial growth factor (VEGF) antibody (at $15 \mathrm{mg} / \mathrm{kg}$ every 3 weeks), or sorafenib (400 mg bid daily). The experimental arm reported a PFS of 6.8 months compared to 4.3 for sorafenib and an OS of 19.2 months compared to 13.4 months in the standard arm. The ORR was higher with the combination treatment, being $27.3 \%$ compared to $11.9 \%$ in patients receiving sorafenib. The combination reported a manageable safety profile, with hypertension being the most common treatment-related adverse event (TRAE). Analysis of patient-reported outcomes in terms of quality of life was superior for atezolizumab plus bevacizumab than sorafenib (median time to deterioration 11.2 months vs. 3.6 months) [23]. The rationale of combining an antiangiogenic agent with an ICPI stems from the known immunosuppressive effect of angiogenic factors. For example, tumor-secreted VEGF is known to promote immunosuppression by increasing immunoregulatory cells and reducing $\mathrm{CD} 8^{+} \mathrm{T}$ cells [24]. Moreover, VEGF causes tumor hypoxia by its direct action on endothelial cells, with consequent PD-L1 expression, which further enhances immune suppression [25].

In HCC, the synergy between ICPIs and antiangiogenics has also been exploited by association of multitargeted TKIs with an anti-PD-1/PD-L1 backbone. The combination of pembrolizumab with lenvatinib, for instance, yielded promising results in the phase Ib Keynote-524 trial [26]. Lenvatinib is a multi-TKI targeting VEGF receptors 1-3, fibroblast growth factor (FGF) receptors 1-4, platelet-derived growth factor receptor-a (PDGFRa), RET, and KIT; it has already been approved as first-line monotherapy for advanced HCC following the results of the noninferiority phase III REFLECT trial [7], where it was showed to be noninferior in terms of OS compared to sorafenib (median OS, 13.6 months with lenvatinib vs. 12.3 months with sorafenib; hazard ratio [HR], 0.92), while providing longer PFS (7.3 vs. 3.6 months; $p<0.0001$ ) and higher ORR (mRECIST: $40.6 \%$ vs. $12.4 \%$; $p<0.0001$ ). The rationale of combining lenvatinib and pembrolizumab derives from the ability of lenvatinib to broadly modulate the immunosuppressive signals within the tumor microenvironment, mainly through its antiangiogenic effect. Moreover, recent data indicate that lenvatinib has direct immunomodulatory properties. In fact, according to preclinical models, lenvatinib has been reported to exert part of its action directly on tumor-infiltrating lymphocytes by reducing Treg differentiation and by promoting PD-L1 ubiquitination and degradation through FGF receptor 4 blockade [27]. Furthermore, results from mice models reported increased early $\mathrm{CD} 8^{+} \mathrm{T}$-cell activity and promising antitumor effect of the combination compared to anti-PD-1 monotherapy [28]. The phase Ib trial testing the association of pembrolizumab (200 mg every 3 weeks) and lenvatinib (body weight $\geq 60 \mathrm{~kg}, 12 \mathrm{mg} ;<60 \mathrm{~kg}, 8 \mathrm{mg}$ daily) on 104 treat- 
ment-naïve patients did not report any dose-limiting toxicity in the dose escalation phase and showed an ORR of $46.0 \%$ per mRECIST and $36.0 \%$ per RECIST v1.1, with a median OS of 22 months [26]. The safety profile reflected the spectrum of AEs typical of the two agents, with the most common being hypertension (36\%), diarrhea (35\%), fatigue $(30 \%)$, decreased appetite $(28 \%)$, and hypothyroidism (25\%). Overall, $67 \%$ of participants reported a grade 3 or higher $\mathrm{AE}$, and again hypertension was the most common, affecting $17 \%$ of patients. The combination of lenvatinib and pembrolizumab has been further assessed for efficacy in the phase III randomized LEAP-002 trial (NCT03713593). LEAP-002 randomized 1:1 750 patients to receive the association of pembrolizumab and lenvatinib following the same schedule as the phase Ib study, as firstline treatment for unresectable HCC. Only patients with preserved liver function (Child-Pugh score A) and without main portal vein invasion were included. OS and PFS are the two coprimary endpoints; the accrual is completed, and results are currently pending.

Atezolizumab plus cabozantinib is another combination that has been explored as first-line treatment for unresectable HCC. Cabozantinib is a multi-TKI targeting VEGF receptors 1-3, TAM kinase family (TYRO3, AXL, and MER), KIT, RET, FLT3, and MET [29]. The rationale of combining cabozantinib and atezolizumab stems from the ability of cabozantinib to modulate both adaptive and innate immune response [30]. In particular, apart from acting on VEGF receptors, cabozantinib reduces the infiltration of immunosuppressive neutrophiles in tumor microenvironment by blocking the interaction between MET and HGF, and its interaction with AXL promotes antigen-presenting cells maturation and enhances expression of MHC class I on tumor cells [31]. Moreover, through the specific inhibitory action on the TAM family, cabozantinib stimulates circulating and tumor-infiltrating $\mathrm{T}$ cells, and it contemporarily reprograms macrophages from M2 to M1 phenotype [32]. Preclinical mice models suggested a synergic increase in antitumoral activity by combining cabozantinib with ICPI [30]. In HCC, the combination of atezolizumab plus cabozantinib is being tested for its efficacy against sorafenib in the phase III randomized COSMIC-312 trial [31]. The trial is randomizing patients 2:1:1 into three arms: cabozantinib (40 mg daily orally) plus atezolizumab 1,200 mg IV once every 3 weeks; sorafenib $400 \mathrm{mg}$ bid; or cabozantinib monotherapy (60 mg daily per os). OS and PFS of the doublet arm versus sorafenib represent the 2 coprimary endpoints, and PFS of single agent versus control is the secondary endpoint. Results from the first interim analysis have re- cently been reported. The combination arm has been shown to reduce the risk of progression by $37 \%$ (HR, 0.63 ; 95\% CI: $0.44-0.91 ; p=0.0012)$, reporting a PFS of 6.8 months, compared to 4.2 with sorafenib and an ORR of $11.2 \%$ [33]. At the same time point, OS was not significantly superior in the combination arm (15.4 vs. 15.5 months, HR 0.90; 95\% CI: 0.69-1.18; $p=0.438)$. Grade 3 or 4 adverse events occurred in $54 \%$ of patients in the combination arm and in $32 \%$ of those receiving sorafenib. The high percentage of patients receiving subsequent lines $(20 \%$ in the combination arm and $37 \%$ in the standard one) could have contributed to the absence of OS advantage; however, longer follow-up is needed to derive definitive conclusions [34].

Another strategy to bolster anticancer immune response is to combine anti-PD-1/PD-L1 antibodies with anti-CTLA-4 agents. This strategy has already demonstrated positive results in several malignancies, including melanoma, NSCLC, renal cell carcinoma, and microsatellite instability-high colorectal cancer [1]. In HCC, the combination of the anti-PD-1 nivolumab plus the antiCTLA-4 ipilimumab has been FDA approved for advanced HCC following sorafenib treatment [35]. The decision was based on the results of the phase I/II CheckMate-040 trial, wherein patients were randomly assigned to receive the combination of nivolumab and ipilimumab at 3 different doses. Patients in arm A, receiving ipilimumab at $3 \mathrm{mg} / \mathrm{kg}$ every 3 weeks in association with nivolumab at $1 \mathrm{mg} / \mathrm{kg}$ for 4 doses followed by nivolumab at $240 \mathrm{mg}$ every 2 weeks, reported the highest ORR (32\%) and OS (22 months vs. 12.5 and 12.7 in arms B and C); results were independent from etiology or PD-L1 status. Cohort A reported the highest rate of any grade adverse events (94\%), with $53 \%$ of participants experiencing grade 3 or higher adverse events and $35 \%$ reporting any grade immune-related adverse event. The follow-on phase III CheckMate 9DW (NCT04039607) is randomizing patients 1:1 to receive nivolumab plus ipilimumab or standard of care (sorafenib or lenvatinib as per investigator choice). The trial is still recruiting, and accrual is planned to be completed in September 2023.

The combination of the anti-PD-L1 durvalumab plus the anti-CTLA-4 tremelimumab is another association of interest in HCC. Results from the phase I/II trial investigating this combination at 4 different doses in patients previously treated with sorafenib have recently been published [20]. The study was made up by three phases. The first one assessed the safety and efficacy of durvalumab (1,500 mg IV every 4 weeks) plus tremelimumab (75 mg IV every 4 weeks); part $2 \mathrm{~A}$ allocated patients 1:1:1 to re-
Fulgenzi/D’Alessio/Talbot/Gennari/ Openshaw/Demirtas/Cortellini/Pinato 
ceive durvalumab monotherapy, or tremelimumab monotherapy, or the combination tested in part 1; part $2 \mathrm{~B}$ assessed the safety of durvalumab (1,500 mg IV every 4 weeks) plus tremelimumab (300 mg IV for 1 single dose); and phase 3 randomized 2:2:1:2 participants to durvalu$\mathrm{mab}$, tremelimumab, or to the combination of both drugs at doses tested in part 1 (tremelimumab $75 \mathrm{mg}$ IV every 4 weeks plus durvalumab 1,500 mg every 4 weeks) and in part 2B (single dose of tremelimumab $300 \mathrm{mg}$ IV plus durvalumab 1,500 mg every 4 weeks). Results from parts $2 \mathrm{~A}$, $2 \mathrm{~B}$, and 3 reported the best outcomes for the combination of tremelimumab (300 mg) plus durvalumab, which showed an ORR of $24 \%$ (compared to $10.6 \%$ for durvalumab monotherapy, $7.2 \%$ for tremelimumab alone, and $9.5 \%$ for tremelimumab at $75 \mathrm{mg}$ plus durvalumab). Similarly, the combination with high dose of tremelimumab (300 mg) yielded the longest median OS (18.5 months vs. 13.6 for durvalumab vs. 15.1 for tremelimumab and 11.5 months for tremelimumab at $75 \mathrm{mg}$ plus durvalumab). The safety profile appeared to be more favorable compared to that described for nivolumab and ipilimumab. Tremelimumab monotherapy resulted in the highest rate of serious TRAEs (24.6\% compared to $17.6 \%$ in the arm of highdose tremelimumab plus durvalumab). Tremelimumab containing regimens were characterized by higher incidence of TRAEs requiring systemic steroids $(24.3 \%$ in combination with tremelimumab 300 , compared to $9.9 \%$ in the case of durvalumab monotherapy). These regimens (durvalumab monotherapy, durvalumab plus tremelimumab 75 or 300 combination) are being compared against sorafenib in the randomized phase III HIMALAYA trial. The study has recently completed the accrual, and final results are still pending (efficacy, tolerability, and biologic activity of a novel regimen of tremelimumab $[\mathrm{T}]$ in combination with durvalumab [D] for patients [pts] with advanced hepatocellular carcinoma [aHCC]). However, it has recently been announced that the association of a high single dose of tremelimumab followed by maintenance therapy with durvalumab has met the primary end point showing a survival advantage over sorafenib, and durvalumab monotherapy has been described to be noninferior to sorafenib (NCT03298451) [36].

In this rapidly evolving landscape, patient selection remains key, and predictive biomarkers are urgently required to allocate patients to the best treatment, avoiding toxicities to those who are not expected to derive therapeutic benefit. Moreover, it remains a major clinical challenge to treat patients generally excluded from clinical trials, particularly those with deranged liver function (ChildPugh score B) or suboptimal performance status [37].

Systemic Treatments for HCC

\section{Predictive Biomarkers of Response}

Despite the recent achievements, there is still a significant percentage of patients who fail to respond to ICPIbased therapies, and therefore predictive biomarkers are required to identify a priori this subset of patients. Moreover, the absence of reliable biomarkers is also, at least in part, responsible for the formal failure of phase III trials testing ICPI monotherapy. Differently from other oncological disease, the expression of PD-L1 by tumor and/or inflammatory cells does not seem to carry a robust predictive significance, and its spatial and temporal heterogeneity, along with the absence of standardized methods for its evaluation, limits its clinical utility $[38,39]$.

Tumor mutational burden (TMB) is a term used to describe the number of nonsynonymous mutations in the genome. It reflects the number of neo-antigens present in neoplastic cells, and it is therefore considered a surrogate of tumor immunogenicity. In other oncological indications, high TMB (defined as $>10$ mutations per megabase) is a predictive marker of response to immunotherapy. However, the prevalence of high TMB in HCC is too low to allow the broad applicability of this marker [40]. Deficiency of mismatch repair mechanisms is a surrogate of hypermutated phenotype, and it is used as an agnostic predictor of response to PD-1 inhibitors; however, it is rarely present in HCC [41].

HCC etiology appears to influence ICPI response; according to a recent study, mice models of NASH-related HCC are intrinsically resistant to anti-PD-1 therapy [42]. Moreover, administration of ICPIs to mice with NASHinduced cirrhosis resulted in increased risk of HCC development. In the same study, data from a meta-analysis including three main ICPIs trials were reported, and they confirmed a reduced benefit in patients affected by nonviral HCC. Based on these data, thorough stratification according to etiology will be necessary in future clinical trials [42]. Other factors that are currently being explored for their potential effect on ICPIs efficacy include gut microbiota [43], concomitant medications [44, 45], and specific genetic traits [46].

\section{Second-Line Treatments}

Following results of the phase II Keynote-224 and CkeckMate-040 trials, pembrolizumab and the combination of nivolumab plus ipilimumab have been FDA approved as second-line treatment after sorafenib therapy. Regorafenib, cabozantinib, and ramucirumab are the only 
drugs tested in phase III trials after sorafenib. Regorafenib, a multi-TKI against VEGFR1-3, PDGFR, FGFR, TIE2, KIT, RET, and RAF, was tested against placebo in the phase III RESORCE trial, and it showed survival advantage in patients who had progressed following sorafenib treatment [47], and patients who were intolerant to sorafenib were not included in the trial. The phase III CELESTIAL placebo-controlled trial tested cabozantinib (60 mg daily) in patients with advanced HCC who had progressed to at least one prior line. Cabozantinib, a multiTKI targeting VEGF receptors 1, 2, and 3, MET, and AXL showed significant superiority versus placebo in terms of both OS (10.2 vs. 8 months) and PFS (5.2 vs. 1.9 months), despite providing an ORR of $4 \%$. Of note, $27 \%$ of patients in the cabozantinib arm had received two previous lines, and the PFS advantage was preserved in this subgroup, even if the OS improvement was not statistically significant [48]. Ramucirumab is a VEGFR-2-directed monoclonal antibody, and it is FDA approved as second-line therapy after sorafenib in patients with baseline value of alpha-fetoprotein $\geq 400 \mathrm{ng} / \mathrm{mL}$. The approval was motivated by the results of the phase III REACH-2 trial [49], which showed survival advantage in the experimental arm against placebo. According to matching-adjusted indirect comparisons, there is no significant difference between second-line options [50]; however, direct comparisons do not exist. Therefore, the choice should be based on the tolerability of previous therapies and on the availability of drugs according to local regulatory agencies. Moreover, with the approval of new front-line treatments, more data are required to define the best sequencing.

\section{Adjuvant/Neoadjuvant Treatments}

The recurrence rate after surgery, OLT, or ablation is as high as $70 \%$ after 5 years [51]. Recurrence can be either due to the re-emergence of microscopic residual disease (early recurrence) or to the development of new tumors in the context of chronic liver inflammation (late recurrence) [52]. To date, the only strategy to prevent the latter is controlling underlying liver disease (e.g., antiviral therapies, lifestyle correction, and alcohol abstention). Effective adjuvant treatments should prevent both early and late recurrences, but unfortunately there are currently no approved adjuvant treatments for HCC [10]. Sorafenib has been tested as adjuvant treatment after resection or ablation in the phase III STORM trial, but it failed to reduce the risk of recurrence while increasing the rate of adverse events [53]. After showing activity in advanced stages, ICPIs, alone
(NCT03383458) (NCT03867084) or in combination with antiangiogenic agents, are being investigated in phase I and II trials after TACE (NCT03397654), resection, or ablation in high-risk patients (NCT04102098) (NCT03847428). The use of adjuvant immunotherapy after OLT remains an open issue, and it is not currently recommended outside clinical trials, due the risk of organ rejection (up to $32 \%$ ) following immune system stimulation [54].

While the aim of adjuvant treatments is to reduce the risk of recurrence, neoadjuvant therapies have the objective to allow curative treatments even in those patients who are diagnosed with unresectable disease and, at the same time, reduce the risk of recurrence after ablation or resection. Currently, there are no standard neoadjuvant treatments in HCC. Locoregional treatments, such as TACE [55] and transarterial radioembolization (TARE), have been proposed in this setting [56]; however, the lack of systemic activity on micrometastasis limits their efficacy. TKIs, given their mainly cytostatic effect, are not able to achieve the adequate tumor shrinkage required in the neoadjuvant setting [52]. Currently, ICPIs are being tested in this setting in phase II trials, both alone (NCT03630640) and as dual checkpoint inhibition [57]. As stated for the adjuvant setting, the use of ICPIs before OLT is currently hampered by the risk of organ rejection, and specific trials addressing this issue are required.

\section{Conclusion}

ICPIs have become an essential therapeutic modality in the treatment landscape of advanced HCC, and the association of ICPIs with antiangiogenic drugs or other ICPIs targeting different pathways has emerged as the most promising strategy to tackle the intrinsic immune resistance of HCC. The promising results in the advanced setting have prompted investigators to test ICPIs in the adjuvant and neoadjuvant settings, wherein previous treatment have failed to provide any benefit. In this rapidly evolving landscape, a number of open issues remain unanswered. The optimal treatment sequence in a scenario where second-line trials post-immunotherapy are lacking is still undefined. Other important challenges are the use of ICPIs before or after OLT and patient selection in the absence of validated predictive biomarkers and the delivery of ICPI in patients classically excluded from clinical trials, like those with impaired liver function, autoimmunity, and HIV infection. Prospective research continues to be required to comprehensively evaluate the expanding role of systemic therapy in HCC. 


\section{Acknowledgment}

The figure has been created with BioRender.com.

\section{Conflict of Interest Statement}

D.J.P. received lecture fees from ViiV Healthcare and Bayer Healthcare and travel expenses from BMS and Bayer Healthcare; consulting fees for Mina Therapeutics, EISAI, Roche, and Astra Zeneca; and received research funding (to institution) from MSD and BMS. A.C. received consulting fees from MSD, BMS, AstraZeneca, and Roche and speakers' fee from AstraZeneca, MSD, Novartis, and Astellas. All remaining authors have declared no conflicts of interest.

\section{Funding Sources}

A.D. is supported by grant funding from the European Association for the Study of the Liver (Andrew Burroughs Fellowship). D.J.P. is supported by grant funding from the Wellcome Trust Strategic Fund (PS3416) and acknowledges grant support from the Cancer Treatment and Research Trust (CTRT) and infrastructural support by the Cancer Research UK Imperial Centre and the NIHR Imperial Biomedical Research Centre. V.E.G. is an employee and shareholder of F. Hoffmann-La Roche, Ltd.

\section{Author Contributions}

Study concept and design: D.J. Pinato. Acquisition of data: all authors. Analysis and interpretation of data: all authors. Drafting of the manuscript: C.A.M. Fulgenzi. Manuscript revision and input: all authors. Statistical analysis: N/A. Obtained funding: D.J. Pinato. Study supervision: D.J. Pinato.

\section{References}

1 Fulgenzi CAM, Talbot T, Murray SM, Silletta M, Vincenzi B, Cortellini A, et al. Immunotherapy in hepatocellular carcinoma. Curr Treat Options Oncol. 2021 Aug 23; 22(10):87.

2 Pinato DJ, Fessas P, Sapisochin G, Marron TU. Perspectives on the neoadjuvant use of immunotherapy in hepatocellular carcinoma. Hepatology. 2021 Jul;74(1):483-90.

3 Galati G, Massimo Vainieri AF, Maria Fulgenzi CA, Di Donato S, Silletta M, Gallo P, et al. Current treatment options for HCC: from pharmacokinetics to efficacy and adverse events in liver cirrhosis. Curr Drug Metab. 2020;21(11):866-84.

4 Yegin EG, Oymaci E, Karatay E, Coker A. Progress in surgical and nonsurgical approaches for hepatocellular carcinoma treatment. Hepatobiliary Pancreat Dis Int. 2016 Jun;15(3):234-56

5 Villanueva A. Hepatocellular carcinoma. N Engl J Med. 2019 Apr 11;380(15):1450-62.

6 Llovet JM, Ricci S, Mazzaferro V, Hilgard P, Gane E, Blanc J-F, et al. Sorafenib in advanced hepatocellular carcinoma. N Engl J Med. 2008 Jul 24;359(4):378-90.

7 Kudo M, Finn RS, Qin S, Han K-H, Ikeda K, Piscaglia F, et al. Lenvatinib versus sorafenib in first-line treatment of patients with unresectable hepatocellular carcinoma: a randomised phase 3 non-inferiority trial. Lancet. 2018 Mar 24;391(10126):1163-73.

8 Yau T, Park JW, Finn RS, Cheng A-L, Mathurin P, Edeline J, et al. CheckMate 459: a randomized, multi-center phase III study of nivolumab (NIVO) vs sorafenib (SOR) as first-line $(1 \mathrm{~L})$ treatment in patients (pts) with advanced hepatocellular carcinoma (aHCC). Ann Oncol. 2019 Oct;30 v874-5.

9 Casak SJ, Donoghue M, Fashoyin-Aje L, Jiang $\mathrm{X}$, Rodriguez L, Shen Y-L, et al. FDA approv- al summary: atezolizumab plus bevacizumab for the treatment of patients with advanced unresectable or metastatic hepatocellular carcinoma. Clin Cancer Res. 2021 Apr 1;27(7): 1836-41.

10 Tovoli F, De Lorenzo S, Trevisani F. Immunotherapy with checkpoint inhibitors for hepatocellular carcinoma: where are we now? Vaccines. 2020 Oct 2;8(4):E578.

11 Bagchi S, Yuan R, Engleman EG. Immune checkpoint inhibitors for the treatment of cancer: clinical impact and mechanisms of response and resistance. Annu Rev Pathol. 2021 Jan 24;16:223-49.

12 Pinato DJ, Guerra N, Fessas P, Murphy R, Mineo T, Mauri FA, et al. Immune-based therapies for hepatocellular carcinoma. Oncogene. 2020 Apr;39(18):3620-37.

13 Pinter M, Jain RK, Duda DG. The current landscape of immune checkpoint blockade in hepatocellular carcinoma a review. JAMA Oncol. 2021 Jan 1 [cited 2021 Dec 13];7(1): 113-23. Available from: https://www.ncbi. nlm.nih.gov/pmc/articles/PMC8265820/pdf/ nihms-1716818.pdf.

14 Llovet JM, Kelley RK, Villanueva A, Singal AG, Pikarsky E, Roayaie S, et al. Hepatocellular carcinoma. Nat Rev Dis Primer. 2021 Jan 21;7(1):6.

15 Sangro B, Gomez-Martin C, de la Mata M, Iñarrairaegui $\mathrm{M}$, Garralda $\mathrm{E}$, Barrera $\mathrm{P}$, et al. A clinical trial of CTLA-4 blockade with tremelimumab in patients with hepatocellular carcinoma and chronic hepatitis C. J Hepatol. $2013 \mathrm{Jul} ; 59(1): 81-8$

16 El-Khoueiry AB, Sangro B, Yau T, Crocenzi TS, Kudo M, Hsu C, et al. Nivolumab in patients with advanced hepatocellular carcinoma (CheckMate 040): an open-label, noncomparative, phase $1 / 2$ dose escalation and expansion trial. Lancet. 2017 Jun 24; 389(10088):2492-502.
17 Zhu AX, Finn RS, Edeline J, Cattan S, Ogasawara S, Palmer D, et al. Pembrolizumab in patients with advanced hepatocellular carcinoma previously treated with sorafenib (KEYNOTE-224): a non-randomised, openlabel phase 2 trial. Lancet Oncol. 2018 Jul; 19(7):940-52.

18 Erck announces KEYTRUDA ${ }^{\star}$ (pembrolizu$\mathrm{mab}$ ) met primary endpoint of overall survival (OS) in patients with advanced hepatocellular carcinoma previously treated with sorafenib, News Release. Merck; 2021 Sep 27.

19 Wainberg ZA, Segal NH, Jaeger D, Lee K-H, Marshall J, Antonia SJ, et al. Safety and clinical activity of durvalumab monotherapy in patients with hepatocellular carcinoma (HCC). J Clin Oncol. 2017 May 20;35(15_Suppl):4071.

20 Kelley RK, Sangro B, Harris W, Ikeda M, Okusaka T, Kang Y-K, et al. Safety, efficacy, and pharmacodynamics of tremelimumab plus durvalumab for patients with unresectable hepatocellular carcinoma: randomized expansion of a phase I/II study. J Clin Oncol. 2021 Sep 20;39(27):2991-3001.

21 Qin S, Finn RS, Kudo M, Meyer T, Vogel A, Ducreux M, et al. RATIONALE 301 study: tislelizumab versus sorafenib as first-line treatment for unresectable hepatocellular carcinoma. Future Oncol. 2019 Jun;15(16):1811-22.

22 Finn RS, Qin S, Ikeda M, Galle PR, Ducreux M, Kim T-Y, et al. Atezolizumab plus bevacizumab in unresectable hepatocellular carcinoma. N Engl J Med. 2020 May 14;382(20): 1894-905.

23 Galle PR, Finn RS, Qin S, Ikeda M, Zhu AX, Kim T-Y, et al. Patient-reported outcomes (PROs) from the Phase III IMbrave150 trial of atezolizumab (atezo) + bevacizumab (bev) vs sorafenib (sor) as first-line treatment (tx) for patients (pts) with unresectable hepatocellular carcinoma (HCC). J Clin Oncol. $2020 \mathrm{Feb}$ 1;38(4_Suppl):476. 
24 Wallin JJ, Bendell JC, Funke R, Sznol M, Korski $\mathrm{K}$, Jones $\mathrm{S}$, et al. Atezolizumab in combination with bevacizumab enhances antigen-specific T-cell migration in metastatic renal cell carcinoma. Nat Commun. 2016 Aug 30;7: 12624.

25 D'Alessio A, Rimassa L, Cortellini A, Pinato DJ. PD-1 blockade for hepatocellular carcinoma: current research and future prospects. J Hepatocell Carcinoma. 2021;8:887-97.

26 Finn RS, Ikeda M, Zhu AX, Sung MW, Baron $\mathrm{AD}$, Kudo M, et al. Phase Ib study of lenvatinib plus pembrolizumab in patients with unresectable hepatocellular carcinoma. J Clin Oncol. 2020 Sep 10;38(26):2960-70.

27 Yi C, Chen L, Lin Z, Liu L, Shao W, Zhang R, et al. Lenvatinib targets FGF receptor 4 to enhance antitumor immune response of antiprogrammed cell death-1 in HCC. Hepatology. 2021 May 26;74(5):2544-60.

28 Kimura T, Kato Y, Ozawa Y, Kodama K, Ito J, Ichikawa $\mathrm{K}$, et al. Immunomodulatory activity of lenvatinib contributes to antitumor activity in the Hepa1-6 hepatocellular carcinoma model. Cancer Sci. 2018 Dec;109(12): 3993-4002.

29 D’Alessio A, Prete MG, Cammarota A, Personeni N, Rimassa L. The role of cabozantinib as a therapeutic option for hepatocellular carcinoma: current landscape and future challenges. J Hepatocell Carcinoma. 2021;8:17791.

30 Bergerot P, Lamb P, Wang E, Pal SK. Cabozantinib in combination with immunotherapy for advanced renal cell carcinoma and urothelial carcinoma: rationale and clinical evidence. Mol Cancer Ther. 2019 Dec;18(12): 2185-93.

31 Kelley RK, Oliver WJ, Hazra S, Benzaghou F, Yau T, Cheng A-L, et al. Cabozantinib in combination with atezolizumab versus sorafenib in treatment-naive advanced hepatocellular carcinoma: COSMIC-312 phase III study design. Future Oncol. 2020 Jul;16(21): 1525-36.

32 Akalu YT, Rothlin CV, Ghosh S. TAM receptor tyrosine kinases as emerging targets of innate immune checkpoint blockade for cancer therapy. Immunol Rev. 2017 Mar;276(1): 165-77.

33 Exelixis and Ipsen announce cabozantinib in combination with an immune checkpoint inhibitor significantly improved progressionfree survival in phase 3 COSMIC-312 pivotal trail in patients with previously untreated advanced liver cancer, News Release. Exelixis, Inc; 2021 Jun 28. Accessed 2021 Jun 28.

34 Kelley RK, Yau T, Cheng A-L, Kaseb A, Qin $\mathrm{S}$, Zhu AX, et al. Cabozantinib (C) plus atezolizumab (A) versus sorafenib (S) as firstline systemic treatment for advanced hepatocellular carcinoma (aHCC): results from the randomized phase 3 COSMIC-312 trial. In: ESMO Asia Virtual. Oncology Week. 2021; VP10-2021.:2021.
35 Yau T, Kang Y-K, Kim T-Y, El-Khoueiry AB, Santoro A, Sangro B, et al. Efficacy and safety of nivolumab plus ipilimumab in patients with advanced hepatocellular carcinoma previously treated with sorafenib: the CheckMate 040 randomized clinical trial. JAMA Oncol. 2020 Nov 1;6(11):e204564.

36 Imfinzi plus tremelimumab significantly improved overall survival in HIMALAYA phase III trial in 1st-line unresectable liver cancer, News Release. AstraZeneca; 2021 Oct 15. Accessed 2021 Oct 15

37 D’Alessio A, Fulgenzi CAM. Treating patients with advanced hepatocellular carcinoma and impaired liver function: broadening the reach of anti-cancer therapy. Liver Cancer Int. 2021 Aug;2(2):31-2.

38 Pinato DJ, Mauri FA, Spina P, Cain O, Siddique A, Goldin R, et al. Clinical implications of heterogeneity in PD-L1 immunohistochemical detection in hepatocellular carcinoma: the Blueprint-HCC study. Br J Cancer. 2019 May;120(11):1033-6.

39 Calderaro J, Rousseau B, Amaddeo G, Mercey $\mathrm{M}$, Charpy C, Costentin C, et al. Programmed death ligand 1 expression in hepatocellular carcinoma: relationship With clinical and pathological features. Hepatology. 2016 Dec; 64(6):2038-46.

40 Ang C, Klempner SJ, Ali SM, Madison R, Ross JS, Severson EA, et al. Prevalence of established and emerging biomarkers of immune checkpoint inhibitor response in advanced hepatocellular carcinoma. Oncotarget. 2019 Jun 18;10(40):4018-25.

41 Yamamoto H, Itoh F, Fukushima H, Kaneto $\mathrm{H}$, Sasaki S, Ohmura T, et al. Infrequent widespread microsatellite instability in hepatocellular carcinomas. Int J Oncol. 2000 Mar;16(3): 543-7.

42 Pfister D, Núñez NG, Pinyol R, Govaere O, Pinter M, Szydlowska M, et al. NASH limits anti-tumour surveillance in immunotherapytreated HCC. Nature. 2021 Apr;592(7854): 450-6.

43 Zheng Y, Wang T, Tu X, Huang Y, Zhang H, Tan D, et al. Gut microbiome affects the response to anti-PD-1 immunotherapy in patients with hepatocellular carcinoma. J Immunother Cancer. 2019 Jul 23;7(1):193.

44 Pinato DJ, Howlett S, Ottaviani D, Urus $\mathrm{H}$, Patel A, Mineo T, et al. Association of prior antibiotic treatment with survival and response to immune checkpoint inhibitor therapy in patients with cancer. JAMA Oncol. 2019 Dec 1;5(12):1774-8.

45 Pinato DJ, Kaseb A, Wang Y, Saeed A, Szafron $\mathrm{D}$, Jun $\mathrm{T}$, et al. Impact of corticosteroid therapy on the outcomes of hepatocellular carcinoma treated with immune checkpoint inhibitor therapy. J Immunother Cancer. 2020 Oct; 8(2):e000726

46 Sia D, Jiao Y, Martinez-Quetglas I, Kuchuk O, Villacorta-Martin C, Castro de Moura M, et al. Identification of an immune-specific class of hepatocellular carcinoma, based on molecular features. Gastroenterology. 2017 Sep; 153(3):812-26.

47 Bruix J, Qin S, Merle P, Granito A, Huang Y-H, Bodoky G, et al. Regorafenib for patients with hepatocellular carcinoma who progressed on sorafenib treatment (RESORCE): a randomised, double-blind, placebo-controlled, phase 3 trial. Lancet. 2017 Jan 7; 389(10064):56-66.

48 Abou-Alfa GK, Meyer T, Cheng A-L, ElKhoueiry AB, Rimassa L, Ryoo B-Y, et al. Cabozantinib in patients with advanced and progressing hepatocellular carcinoma. $\mathrm{N}$ Engl J Med. 2018 Jul 5;379(1):54-63.

49 Zhu AX, Kang Y-K, Yen C-J, Finn RS, Galle PR, Llovet JM, et al. Ramucirumab after sorafenib in patients with advanced hepatocellular carcinoma and increased a-fetoprotein concentrations (REACH-2): a randomised, double-blind, placebo-controlled, phase 3 trial. Lancet Oncol. 2019 Feb; 20(2):282-96.

50 Solimando AG, Susca N, Argentiero A, Brunetti $\mathrm{O}$, Leone $\mathrm{P}$, De Re V, et al. Second-line treatments for advanced hepatocellular carcinoma: a systematic review and bayesian network meta-analysis. Clin Exp Med. 2021 Jun 19. Epub ahead of print.

51 Colecchia A, Schiumerini R, Cucchetti A, Cescon M, Taddia M, Marasco G, et al. Prognostic factors for hepatocellular carcinoma recurrence. World J Gastroenterol. 2014 May 28;20(20):5935-50.

52 Akateh C, Black SM, Conteh L, Miller ED, Noonan A, Elliott E, et al. Neoadjuvant and adjuvant treatment strategies for hepatocellular carcinoma. World J Gastroenterol. 2019 Jul 28;25(28):3704-21.

53 Bruix J, Takayama T, Mazzaferro V, Chau G-Y, Yang J, Kudo M, et al. Adjuvant sorafenib for hepatocellular carcinoma after resection or ablation (STORM): a phase 3, randomised, double-blind, placebo-controlled trial. Lancet Oncol. 2015 Oct;16(13):1344-54.

$54 \mathrm{Au} \mathrm{KP}$, Chok KSH. Immunotherapy after liver transplantation: where are we now? World J Gastrointest Surg. 2021 Oct 27;13(10):126778.

55 Kang W-H, Hwang S, Song G-W, Lee Y-J, Kim K-H, Ahn C-S, et al. Prognostic effect of transarterial chemoembolization-induced complete pathological response in patients undergoing liver resection and transplantation for hepatocellular carcinoma. Liver Transpl. 2017 Jun;23(6):781-90.

56 Toso C, Mentha G, Kneteman NM, Majno P. The place of downstaging for hepatocellular carcinoma. J Hepatol. 2010 Jun;52(6):930-6.

57 Pinato DJ, Cortellini A, Sukumaran A, Cole T, Pai M, Habib N, et al. PRIME-HCC: phase Ib study of neoadjuvant ipilimumab and nivolumab prior to liver resection for hepatocellular carcinoma. BMC Cancer. 2021 Mar 23;21(1):301. 